\title{
summary
}

\section{Fluoride-releasing elastomerics reduce enamel decalcification during fixed appliance therapy}

Banks PA, Chadwick SM, Ascher-Mcdade C, Wright JL. Fluoride releasing elastomerics - a prospective controlled clinical trial. Eur J Orthodont 2000; 22:401-407

Objective To determine whether fluoride-releasing elastomerics reduce enamel decalcification significantly during fixed appliance therapy.

Design Randomised controlled trial.

Intervention Stannous fluoride-releasing modules and chain in test group. Test and control elastomerics changes were made 4-6-weekly.

Outcome measures All patients were scored by a single clinician for decalcification using an enamel decalcification index. Reproducibility of the index was tested using Kappa.

Results See Table 1.

Conclusions Fluoride-releasing elastomerics appear to offer a clinically worthwhile reduction in enamel decalcification during fixed appliance therapy when changed at each treatment visit.
Table 1 Enamel decalcification in control and treated groups

\begin{tabular}{lcc}
\hline & $\begin{array}{c}\text { Fluoride-releasing } \\
\text { elastomerics }\end{array}$ & Control \\
\hline Patients $(\mathrm{n})$ & 45 & 49 \\
Enamel decalcification (\%) & 26 & 73 \\
ARR (\%) & 47 & \\
NNT & $2(95 \% \mathrm{Cl}, 2-3)$ & \\
\hline
\end{tabular}

$\mathrm{ARR}$, absolute risk reduction; NNT, number needed to treat, $\mathrm{Cl}$, confidence interval.

\section{Commentary}

Enamel demineralisation and the need for patient co-operation in its prevention has been a continual challenge for the orthodontist. This paper seems to address both these issues directly and has interesting and clinically relevant outcomes. The authors have focussed on the value of long duration low-dose fluoride release in preventing enamel damage using fluoride-releasing elastomerics in full-banded therapy. A review of most of the other relevant modalities of preventing enamel decalcification emphasises the clinical relevance of this randomised controlled clinical trial. Well-defined inclusion criteria, blinding procedures and a meticulous statistical design contribute to the definitive outcome of this trial. The authors have ensured a pretreatment equivalence and matching of the two groups for relevant variables. The outcome of the trial indicates a reduction of both incidence as well as the severity of decalcification. The overall $47 \%$ reduction in decalcification scores in comparison with the control group is clinically significant and well worth the effort. The results also support the finding of some previous in vitro studies that low dosage prolonged fluoride release from elastomerics would provide significant enamel protection in full-banded therapy. Although fluoride-releasing elastomerics show a rapid deterioration in the oral environment, there was no prolongation of treatment time. The authors indicate only a minor disadvantage of the fluoride-impregnated elastomerics being less elastic and more prone to discoloration and swelling. Fluoride-impregnated elastomerics certainly have a clinically significant role in reducing enamel decalcification in fixed bonded orthodontics.

Non-aesthetic white-spot lesions or larger unattractive areas of decalcification around orthodontic brackets remain a significant problem during fixed-appliance treatment. These areas of demineralisation of enamel around orthodontic attachments usually oc- cur after a period of 1 month. Stannous fluoride-releasing elastomeric modules and chain may provide additional protection against enamel decalcification through fluoride release. This study supports the use of fluoride-releasing elastomerics and is probably the first of its kind to prove the clinical efficacy of these fluoride-releasing elastomerics.

The authors are to be complimented for having studied a vexing but clinically-relevant problem and providing some effective outcomes which have a direct relevance to clinical practice. More such clinically-oriented trials with relevant outcomes would help to resolve the problem of 'intellectual poverty amidst plenty' which assails orthodontic research and literature. It would also emphasise the role of evidence in day-to-day clinical practice.

\section{Anmol S Kalha \\ Department of Orthodontics, College of Dental Sciences, Davangere, India}

